\title{
$N{ }^{\omega}$-Nitro- $\boldsymbol{N}^{\omega}{ }^{\prime}$-Substituted Guanidines: A Simple Class of Nitric Oxide Synthase Inhibitors
}

\author{
Christophe D. Guillon ${ }^{1}$, David D. Wisnoski ${ }^{1}$, Jaya Saxena ${ }^{1}$, Ned D. Heindel ${ }^{1}$, Diane E. Heck ${ }^{2}$, \\ Donald J. Wolff3 ${ }^{3}$ Jeffrey D. Laskin ${ }^{4}$ \\ ${ }^{1}$ Department of Chemistry, Lehigh University, Bethlehem, USA \\ ${ }^{2}$ Department of Environmental Health Science, New York Medical College, Valhalla, USA \\ ${ }^{3}$ Department of Pharmacology, Rutgers University-Robert Wood Johnson Medical School, Piscataway, USA \\ ${ }^{4}$ Department of Environmental and Occupational Medicine, Rutgers University-Robert Wood Johnson \\ Medical School, Piscataway, USA \\ Email: chg3@lehigh.edu
}

Received 27 March 2014; revised 20 April 2014; accepted 28 April 2014

Copyright (C) 2014 by authors and Scientific Research Publishing Inc.

This work is licensed under the Creative Commons Attribution International License (CC BY).

http://creativecommons.org/licenses/by/4.0/

(c) (i) Open Access

\begin{abstract}
A series of $\mathrm{N}^{\omega}$-nitro- $\mathrm{N}^{\omega^{\prime}}$-substituted guanidines has been prepared as potential inhibitors of the human Nitric Oxide Synthase (NOS) isoforms. The reported utility of amino-guanidine and nitroarginine in iNOS inhibition points to a potential similar utility for analogs of nitro-guanidine. The compound library was tested against the three isoforms of Nitric Oxide Synthase (eNOS, iNOS and nNOS). Several candidates showed excellent activity and good selectivity for nNOS. One particular compound even demonstrated good selectivity for iNOS. The potential usefulness of such selective inhibitors is discussed.
\end{abstract}

Keywords

Nitro-Guanidines, Nitric Oxide Synthase (NOS), Isoforms, Selective Inhibitors

\section{Introduction}

Nitric oxide (NO) is a key messenger involved in a wide range of biochemical processes [1]. Its role is crucial for a number of physiological functions and many pathologies can be related to its inadequate release or overproduction [2] [3]. NO is produced from the oxidation of L-arginine to citrulline [4]. The family of enzymes that catalyze this process is called Nitric Oxide Synthase (NOS) [5] and three different isoforms exist [6]. Two are 
constitutive forms, eNOS (e for endothelial, also known as NOS III) involved in the regulation of smooth muscle relaxation, blood pressure and inhibition of platelet aggregation [7]; and nNOS (n for neuronal, also known as NOS I), related to neurotransmission and long-term potentiation [8]. The other isoform iNOS (i for induced, also known as NOS II) is involved in regulation of the immune system and inflammatory responses [9]. These three isoforms have unique roles in separate tissues thus making selective inhibition of either form a suitable strategy for the treatment of specific pathologies. Substantial drug development has been carried out to achieve specific inhibition of the nNOS form as a way to treat strokes and of iNOS for the treatment of septic shock and arthritis. The eNOS form, because of its important role in blood flow regulation, is seldom a clinical therapeutic target. This research has led to the discovery of a number of iNOS and to several nNOS selective inhibitors [1].

However, there is a continuing need for new potent and selective inhibitors of either form of the enzyme as diverse pathologies are found to be linked to these enzymes. Recent work in our laboratories has shown that iNOS inhibitors, such as aminoguanidine $(\mathrm{KI}=830 \mu \mathrm{M})$, attenuate inflammation in the rat lung induced by the toxic vesicant, nitrogen mustard [10]. In fact, aminoguanidine abrogated nitrogen mustard-induced injury and oxidative stress and inflammation at $1 \mathrm{~d}$ and $3 \mathrm{~d}$ post exposure [11].

This finding prompted us to synthesize nitro-guanidine derivatives that displayed increased activity towards iNOS, and indeed two compounds were identified (e.g., compounds 12 and 4). Unexpectedly, most of the other compounds in this library were better nNOS and/or eNOS inhibitors. Although these latter candidates may not be selective for iNOS, they may still suppress the toxicity mediated by iNOS. Moreover, the fact that we have been able to identify nitro-guanidines which are selective for nNOS or eNOS suggests that these may be useful for pathological conditions where suppressing these isoforms of NOS may be beneficial. Other workers are pursuing selective nNOS inhibitors for therapeutic intervention in neuromuscular disorders [12] and neurodegenerative pathologies such as Alzheimer's and Parkinson's disease [13] [14]. A number of those known inhibitors are analogs of the substrate L-arginine and include, in particular, N-nitro-arginine [15] [16]. Some of them achieved not only good activity but also demonstrated excellent iNOS selectivity. Our work focused on the preparation of non-amino-acid guanidine-based analogs of $\mathrm{N}^{\omega}$-nitro-arginine which has been reported to have about a 250-fold selectivity for nNOS versus iNOS [14].

\section{Discussion and Results}

\subsection{Chemistry}

All the compounds prepared were synthesized through a pathway adapted from a process originally developed for the preparation of nitro-guanidines as potential fertilizers [17]-[19]. In such an approach, a commercially available reactive nitroso compound (1-methyl-3-nitro-1-nitrosoguanidine) was used to prepare the target products, as solids, in a convenient one step reaction (Scheme 1). In general, the yields ranged from $33 \%$ to $95 \%$ and for most compounds were usually greater than $60 \%$. The details of the compounds synthesized are presented in Table 1 . When the purity of the crude compounds did not prove satisfactory, as assessed by ${ }^{1} \mathrm{H}$ NMR, they were easily crystallized from a number of solvents such as methanol or methanol/chloroform. Low yields $(<20 \%)$ were obtained when the reacting amines were poor nucleophiles [15] [16] but the compounds could nonetheless be isolated in sufficient amounts to be tested in the NOS assays even in those cases. The purity of all those compounds was assessed by conventional analytical methods to be greater than $98 \%$.

\subsection{Biology}

Once isolated and characterized, all the compounds were submitted to NOS screening for which the isolated isoforms of the enzyme were used (iNOS, nNOS and eNOS). The data obtained is summarized in Table 2. Compounds 4, 8, 12, 13, 14, 15, 17, 18 and 27 demonstrated single digit micromolar activities against one or<smiles>[R1]N[R2]</smiles> 
Table 1. $N^{\omega}$-nitro- $N^{\omega^{\prime}}$-substituted guanidines synthesized according to Scheme 1.

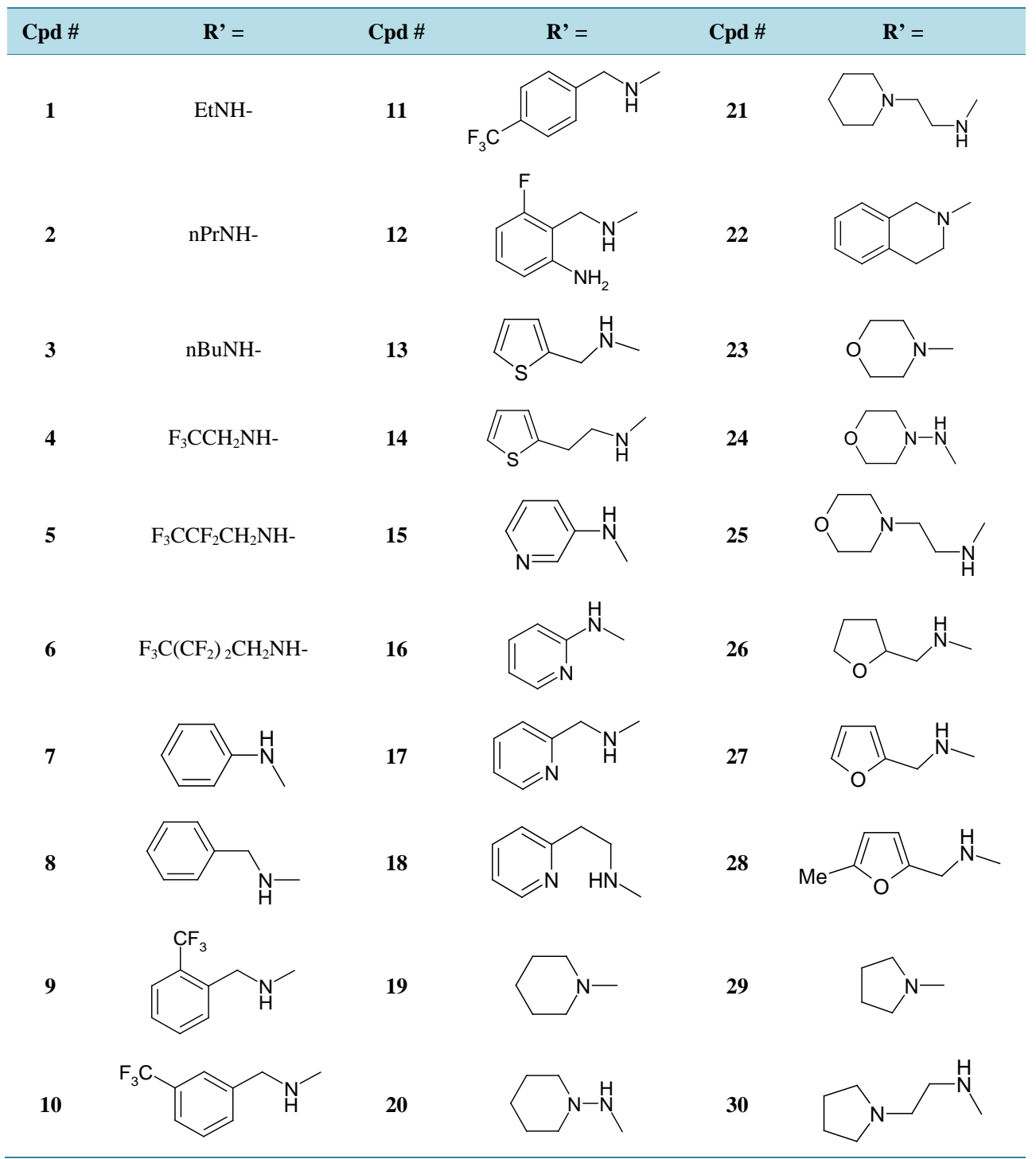

several of the NOS isoforms. In particular 1-nitro-3- (pyridin-3-yl) guanidine (15) proved active against both eNOS $(0.3 \mu \mathrm{M})$ and nNOS $(0.5 \mu \mathrm{M})$. As far as selectivity is concerned, we were able to identify a number of compounds displaying nNOS selectivity. One such compound, 1- ((5-methylthiophen-2-yl)methyl)-3-nitroguanidine (28), although of marginal potency, nevertheless showed selectivity toward nNOS (eNOS/nNOS = 35). It is to be noted that the same compound also demonstrated nNOS over iNOS selectivity (iNOS/nNOS = 30). To a smaller extent, compounds $\mathbf{9}$ and $\mathbf{1 0}$ also showed nNOS selectivity with eNOS to nNOS ratios of 15 and 13 respectively. Although it was not the primary focus of this study, we also herein report some impressive nNOS over iNOS selectivity with compounds $\mathbf{2}, \mathbf{1 3}$ and $\mathbf{1 5}$ displaying iNOS to nNOS ratio of 62, 50 and 100 respectively. Compound 4, which had the lowest across-the-board inhibition $\mathrm{IC}_{50}$ 's for all three NOS isoforms (see Table 2), was tested topically for inflammation suppression in a standard mustard-induced inflamed mouse ear vesicant model [20]. In this assay 4 showed 41\% suppression of inflammation compared to classic anti-inflammatory standards such as S-naproxen (11\%), diclofenac (17\%), indomethacin (46\%), menthol (53\%), or farnesol (64\%) [21]. These simple guanidine mimics of the natural substrate of the enzyme, L-arginine, demonstrate that the amino acid portion of that substrate is not required to achieve activity and even selectivity toward the nNOS isoform. Guanidines can be considered a potential platform from which a more in depth SAR could be built and one that could yield even better inhibitors. 
Table 2. In vitro activity and selectivity of nitro-guanidines against the isolated isoforms of NOS.

\begin{tabular}{|c|c|c|c|c|c|}
\hline \multirow{2}{*}{ Compound } & \multicolumn{5}{|c|}{$\mathrm{IC}_{50}(\mu \mathrm{M})$} \\
\hline & nNOS & eNOS & iNOS & e/n ratio & $\mathrm{i} / \mathbf{n}$ ratio \\
\hline $\mathrm{H}_{2} \mathrm{~N}-\mathrm{C}(=\mathrm{NH})-\mathrm{NH}-\mathrm{NO}_{2}{ }^{(*)}$ & 250 & 110 & 1022 & 0.4 & 4 \\
\hline $\mathrm{MeHN}-\mathrm{C}(=\mathrm{NH})-\mathrm{NH}-\mathrm{NO}_{2}{ }^{\left({ }^{*}\right)}$ & 200 & 51 & 5680 & 0.3 & 28 \\
\hline 1 & 497 & 42 & $>2000$ & 0.1 & $\mathrm{~N}-\mathrm{A}$ \\
\hline 2 & 25 & 47 & 1540 & 2 & 62 \\
\hline 3 & 45 & 137 & 600 & 3 & 13 \\
\hline 4 & 5.3 & 1.4 & 30 & 0.3 & 6 \\
\hline 5 & 80 & 54 & 200 & 0.7 & 2.5 \\
\hline 6 & 489 & $>2000$ & $>2000$ & $\mathrm{~N}-\mathrm{A}$ & N-A \\
\hline 8 & 45 & 3 & 600 & 0.1 & 13 \\
\hline 9 & 16 & 244 & 170 & 15 & 11 \\
\hline 10 & 38 & 477 & 1700 & 13 & 45 \\
\hline 11 & 112 & 227 & 542 & 2 & 5 \\
\hline 12 & $>1000$ & 125 & 6.7 & N-A & N-A \\
\hline 13 & 18 & 0.94 & 902 & 0.1 & 50 \\
\hline 14 & 51 & 3.4 & 815 & 0.7 & 16 \\
\hline 15 & 0.5 & 0.3 & 55 & 0.6 & 110 \\
\hline 16 & 79 & 13 & 667 & 0.2 & 8 \\
\hline 17 & 49 & 0.67 & 314 & 0.01 & 6 \\
\hline 18 & 67 & 8.3 & 1350 & 0.1 & 20 \\
\hline 19 & 460 & 1510 & 1800 & 3 & 4 \\
\hline 20 & $>2000$ & $>2000$ & $>2000$ & N-A & N-A \\
\hline 21 & 790 & 644 & $>2000$ & 0.8 & N-A \\
\hline 22 & $>1000$ & 700 & 690 & N-A & N-A \\
\hline 23 & $>2000$ & $>2000$ & $>2000$ & N-A & N-A \\
\hline 24 & 1590 & $>2000$ & $>2000$ & N-A & N-A \\
\hline 25 & $>2000$ & $>2000$ & $>2000$ & $\mathrm{~N}-\mathrm{A}$ & N-A \\
\hline 26 & $>2000$ & 1005 & $>2000$ & $\mathrm{~N}-\mathrm{A}$ & N-A \\
\hline 27 & 8.3 & 17 & 236 & 2 & 28 \\
\hline 28 & 22 & 780 & 667 & 35 & 30 \\
\hline 29 & $>2000$ & $>2000$ & $>2000$ & N-A & N-A \\
\hline 30 & 167 & 555 & 667 & 4 & 4 \\
\hline
\end{tabular}

$\left({ }^{*}\right)$ : obtained from commercial sources.

\section{Conclusion}

We have prepared a series of $\mathrm{N}^{\omega}$-nitro- $\mathrm{N}^{\omega^{\prime}}$-substituted guanidines in a convenient one step reaction. Evaluation of their inhibitory activity against the isoforms of NOS led to the identification of a number of hits with micromolar and one (15) with sub-micromolar potency. Among those hits, several also demonstrated selectivity toward nNOS (9, 10, 28 nNOS over eNOS selectivity and 2, 13, 15 and 28 for nNOS over iNOS selectivity). The 
most promising compound of this family (15) could be considered a lead candidate for further development of potent nNOS inhibitors in the class. As potential iNOS inhibitors for use in our mustard-induced lung damage model, $4, \mathbf{5}, \mathbf{9}, \mathbf{1 2}$, and 15 were substantially more potent than aminoguanidine with 12 having the best margin of safety for minimal cross reactivity with nNOS and eNOS. When comparing the activities of the pyridine-containing compounds in this set, i.e., 15, 16, 17, and 18, it is compound, 15 for which external hydrogen-bonding (both $\mathrm{H}$-donor and $\mathrm{H}$-acceptor) is the most probable, which has the greatest inhibition of all three isoforms. Recent crystal structure studies have claimed that precisely such external hydrogen bonding by twisted 2-aminopyridines makes these molecules important pharmacophores in inhibition of nNOS and eNOS [22].

\section{Experimental Section}

\subsection{Chemistry}

${ }^{1} \mathrm{H}$ NMR spectra were recorded at $360 \mathrm{MHz}$ and $500 \mathrm{MHz}$ on a Bruker AMX-360 and DRX-500 spectrometer respectively. Chemical shifts were measured relative to $\mathrm{CDCl}_{3}(\delta=7.24), \mathrm{CD}_{3} \mathrm{OD}(\delta=3.33)$ or acetone-d6 $(\delta=$ 2.04) for ${ }^{1} \mathrm{H}$ and expressed indirectly in relation to TMS. The following abbreviations are used to describe the signal multiplicity: s (singulet), d (doublet), t (triplet), q (quadruplet) and m (multiplet). Chemical shifts are expressed in ppm and listed as follow: shift in ppm (multiplicity, coupling constant, and attribution). IR Spectra were recorded on a Mattson Polaris FT-IR spectrophotometer as $\mathrm{NaCl}$ discs for the crystalline samples. Thin-layer chromatography (TLC) were performed with plates $(0.25 \mathrm{~mm})$ pre-coated with fluorescent silica gel. Reaction components were then visualized under UV light and/or with iodine and/or with a saturated solution of $\mathrm{KMnO}_{4}$ in aqueous $\mathrm{NaOH}(1 \mathrm{~N})$. Silica gel (230 - 400 mesh) was used for flash chromatography separations. Uncorrected melting points (mp) were determined with a Thomas Hoover capillary melting point apparatus. Combustion analyses were provided by Intertek, Whitehouse, NJ.

1-ethyl-3-nitroguanidine (1):

Ethylamine $(0.235 \mathrm{~mL}, 3.60 \mathrm{mmol})$ was added dropwise, at $10^{\circ} \mathrm{C}$, to a suspension of 1-methyl-3-nitro-1-nitrosoguanidine $(529 \mathrm{mg}, 3.60 \mathrm{mmol})$ in a mixture of ethanol and water $(50 / 50, \mathrm{v} / \mathrm{v}, 8 \mathrm{~mL})$. After $24 \mathrm{~h}$ at room temperature, the reaction mixture was quenched by addition of $10 \mathrm{~mL}$ of $\mathrm{NaOH}(1 \mathrm{~N})$ and $10 \mathrm{~mL}$ of saturated aqueous sodium chloride. This phase was extracted 5 times with chloroform and after the usual work-up the evaporation of the organic layer afforded (209 $\mathrm{mg}, 44 \%)$ of 1 as a white solid, $\mathrm{mp}=149^{\circ} \mathrm{C}-150^{\circ} \mathrm{C}$. IR $(\mathrm{KBr})$ : 1609, 1698, 3126, 3225, 3481. ${ }^{1} \mathrm{H}$ NMR $\left(\mathrm{CD}_{3} \mathrm{OD}\right) \delta: 1.21\left(\mathrm{t},{ }^{3} \mathrm{~J}=6.7 \mathrm{~Hz}, \mathrm{CH}_{3}\right) ; 3.28\left(\mathrm{t},{ }^{3} \mathrm{~J}=6.8 \mathrm{~Hz}, \mathrm{CH}_{2}\right)$. Anal.Calcd.for $\mathrm{C}_{3} \mathrm{H}_{8} \mathrm{~N}_{4} \mathrm{O}_{2}$ : C, 27.27; H, 6.10; N, 42.41. Found: C, 27.13; H, 5.78; N, 42.15.

1-nitro-3-propylguanidine (2):

The title compound was prepared according to the above procedure using propylamine $(0.32 \mathrm{~mL}, 3.89 \mathrm{mmol})$ and 1-methyl-3-nitro-1-nitrosoguanidine (498 mg, $3.39 \mathrm{mmol})$. The reaction afforded (330 $\mathrm{mg}, 67 \%)$ of 2 as a white solid. $\mathrm{mp}=97^{\circ} \mathrm{C}-98^{\circ} \mathrm{C}$. IR $(\mathrm{KBr}): 1600,3163,3310,3388 .{ }^{1} \mathbf{H}$ NMR $\left(\mathrm{CD}_{3} \mathrm{OD}\right) \delta: 0.97\left(\mathrm{t},{ }^{3} \mathrm{~J}=7.3 \mathrm{~Hz}\right.$, $\left.\mathrm{CH}_{3}\right)$; 1.56-1.66 (m, $\left.\mathrm{CH}_{2 \beta}\right)$; $3.19\left(\mathrm{t},{ }^{3} \mathrm{~J}=7.1 \mathrm{~Hz}, \mathrm{CH}_{2 \alpha}\right.$ ). Anal.Calcd.for $\mathrm{C}_{4} \mathrm{H}_{10} \mathrm{~N}_{4} \mathrm{O}_{2}$ : C, 32.87; H, 6.90; N, 38.34 . Found: C, 32.88; H, 6.74; N, 38.63.

1-butyl-3-nitroguanidine (3):

Butylamine $(0.35 \mathrm{~mL}, 3.50 \mathrm{mmol})$ was added dropwise to a suspension of 1-methyl-3-nitro-1-nitrosoguanidine $(501 \mathrm{mg}, 3.41 \mathrm{mmol})$ in a mixture of ethanol and water $(50 / 50, \mathrm{v} / \mathrm{v}, 13 \mathrm{~mL})$. After $24 \mathrm{~h}$ at room temperature, the product that precipitated out of solution was isolated by suction filtration, washed with cold water and dried with the assistance of $\mathrm{P}_{2} \mathrm{O}_{5}$ affording 5 (258 mg) as a white solid. An additional fraction (210 $\left.\mathrm{mg}\right)$ of 5 was isolated after extraction with chloroform of the remaining aqueous layer quenched with $10 \mathrm{~mL}$ of saturated aqueous sodium chloride. Overall (468 mg, 86\%) of 3 were isolated. It was purified by crystallization from a mixture $\mathrm{Et}_{2} \mathrm{O}$ and $\mathrm{CHCl}_{3}$ to afford a white solid. $\mathrm{mp}=82^{\circ} \mathrm{C}-83^{\circ} \mathrm{C}$. IR (KBr): 1552, 1603, 1651, 3165, 3309, 3391. ${ }^{1} \mathbf{H}$ NMR $\left(\mathrm{CD}_{3} \mathrm{OD}\right) \delta: 0.96\left(\mathrm{t},{ }^{3} \mathrm{~J}=7.3 \mathrm{~Hz}, \mathrm{CH}_{3}\right) ; 1.34-1.45\left(\mathrm{~m}, \mathrm{CH}_{2 \gamma}\right) ; 1.5-1.64\left(\mathrm{~m}, \mathrm{CH}_{2 \beta}\right) ; 3.22\left(\mathrm{t},{ }^{3} \mathrm{~J}=7.1 \mathrm{~Hz}\right.$, $\mathrm{CH}_{2 \alpha}$ ). Anal.Calcd.for $\mathrm{C}_{5} \mathrm{H}_{12} \mathrm{~N}_{4} \mathrm{O}_{2}$ : C, 37.49; H, 7.55; N, 34.98. Found: C, 37.35; H, 7.30; N, 34.88.

1-nitro-3-(2,2,2-trifluoroethyl)guanidine (4):

2,2,2-trifluoroethylamine $(1 \mathrm{~g}, 10.09 \mathrm{mmol})$ was added dropwise, at $5^{\circ} \mathrm{C}$, to a suspension of 1-methyl-3-nitro1-nitrosoguanidine $(886 \mathrm{mg}, 6.02 \mathrm{mmol})$ in a mixture of ethanol and water $(50 / 50, \mathrm{v} / \mathrm{v}, 14 \mathrm{~mL})$. After $1 \mathrm{~h}$ the mixture was reacted at $55^{\circ} \mathrm{C}$ for $4 \mathrm{~h}$ and overnight at room temperature. Overtime the mixture turned to a clear pale yellow solution. On cooling down with an ice bath the product precipitated out of solution and was isolated by suction filtration, washed with cold water and dried with the assistance of $\mathrm{P}_{2} \mathrm{O}_{5}$ affording $4(822 \mathrm{mg}, 65 \%)$ as 
a white solid. $\mathrm{mp}=145.5^{\circ} \mathrm{C}-146.5^{\circ} \mathrm{C}$. IR (KBr): 1562, 1600, 1642, 3122, 3246, 3398. ${ }^{1} \mathbf{H}$ NMR (CD NDD $_{3} \delta$ : $4.02\left(\mathrm{q},{ }^{3} \mathrm{~J}_{\mathrm{H}, \mathrm{F}}=9.05 \mathrm{~Hz}, \mathrm{CH}_{2}\right.$ ). Anal.Calcd.for $\mathrm{C}_{3} \mathrm{H}_{5} \mathrm{~F}_{3} \mathrm{~N}_{4} \mathrm{O}_{2}$ : C, 19.36; H, 2.71; N, 30.11. Found: C, 19.77; H, $2.40 ; \mathrm{N}, 30.01$.

1-nitro-3-(2,2,3,3,3-pentafluoropropyl)guanidine (5):

2,2,3,3,3-pentafluoropropylamine $(0.37 \mathrm{~mL}, 3.48 \mathrm{mmol})$ was added dropwise to a suspension of 1-methyl-3nitro-1-nitrosoguanidine (469 mg, $3.17 \mathrm{mmol}$ ) in a mixture of ethanol and water (50/50, v/v, $14 \mathrm{~mL}$ ). The yellow 1-methyl-3-nitro-1-nitrosoguanidine went rapidly in solution and overtime a white precipitate formed, the remaining solution being colorless. After $20 \mathrm{~h}$, the solid was collected by suction filtration, washed with cold water and dried in vacuo with the assistance of $\mathrm{P}_{2} \mathrm{O}_{5}$ affording (688 $\mathrm{mg}, 91 \%$ ) of 5 as a white solid. mp = $158.5^{\circ} \mathrm{C}-159^{\circ} \mathrm{C} . \mathbf{I R}(\mathrm{KBr}): 1534,1651,3307,3426 .{ }^{1} \mathbf{H} \mathbf{N M R}\left(\mathrm{CD}_{3} \mathrm{OD}\right) \delta: 4.02\left(\mathrm{t},{ }^{3} \mathrm{~J}_{\mathrm{H}, \mathrm{F}}=15.0 \mathrm{~Hz}, \mathrm{CH}_{2}\right)$. Anal.Calcd.for $\mathrm{C}_{4} \mathrm{H}_{5} \mathrm{~F}_{5} \mathrm{~N}_{4} \mathrm{O}_{2}$ : C, 20.35; H, 2.13; N, 23.73. Found: C, 20.66; H, 2.01; N, 23.84.

1-(2,2,3,3,3,4,4,4-heptafluorobutyl)-2-nitro-guanidine (6):

The title compound was prepared according to the above procedure using 2,2,3,3,3,4,4,4-heptafluorobutylamine (0.63 g, $2.85 \mathrm{mmol}$ ) and 1-methyl-3-nitro-1-nitrosoguanidine (419 mg, $3.39 \mathrm{mmol}$ ) in a mixture of ethanol and water $(50 / 50, \mathrm{v} / \mathrm{v}, 14 \mathrm{~mL})$. The reaction afforded $(530 \mathrm{mg}, 65 \%)$ of 6 as a white solid. $\mathrm{mp}=127.5^{\circ} \mathrm{C}-$ 128.5 ${ }^{\circ}$ C. IR (KBr): 1609, 1663, 3172, 3324, 3423. ${ }^{1} \mathbf{H}$ NMR (CD $\left.{ }_{3} \mathrm{OD}\right) \delta: 4.14\left(\mathrm{t},{ }^{3} \mathrm{~J}_{\mathrm{H}, \mathrm{F}}=15.5 \mathrm{~Hz}, \mathrm{CH}_{2}\right)$. Anal.Calcd.for $\mathrm{C}_{5} \mathrm{H}_{5} \mathrm{~F}_{7} \mathrm{~N}_{4} \mathrm{O}_{2}$ : C, 20.99; H, 1.76; N, 19.58. Found: C, 20.86; H, 1.62; N, 19.82.

1-nitro-3-phenylguanidine (7):

The title compound was prepared according to the above procedure using aniline $(0.31 \mathrm{~mL}, 3.39 \mathrm{mmol})$ and 1-methyl-3-nitro-1-nitrosoguanidine (499 mg, $3.39 \mathrm{mmol})$ in a mixture of ethanol and water (50/50, v/v, $14 \mathrm{~mL})$. The reaction afforded (477 $\mathrm{mg}, 78 \%$ ) of 7 as a white solid. $\mathrm{mp}=155^{\circ} \mathrm{C}-156.5^{\circ} \mathrm{C} \mathrm{IR}(\mathrm{KBr}): 1570,1645,3150$, 3378. ${ }^{1} \mathrm{H}$ NMR $\left(\mathrm{CD}_{3} \mathrm{OD}\right) \delta$ : 7.30 - 7.51 (m, $\left.\mathrm{CH}=\right)$. Anal.Calcd.for $\mathrm{C}_{7} \mathrm{H}_{8} \mathrm{~N}_{4} \mathrm{O}_{2}+0.25 \mathrm{H}_{2} \mathrm{O}: \mathrm{C}, 45.53 ; \mathrm{H}, 4.64 ; \mathrm{N}$, 30.34. Found: C, 45.49; H, 4.64; N, 30.34.

1-benzyl-3-nitroguanidine (8):

The title compound was prepared according to the above procedure using benzylamine $(0.40 \mathrm{~mL}, 3.65 \mathrm{mmol})$ and 1-methyl-3-nitro-1-nitrosoguanidine $(533.5 \mathrm{mg}, 3.62 \mathrm{mmol})$ in a mixture of ethanol and water $(50 / 50, \mathrm{v} / \mathrm{v}$, $14 \mathrm{~mL})$. The reaction afforded (628 $\mathrm{mg}, 95 \%)$ of 8 as a white solid. $\mathrm{mp}=181^{\circ} \mathrm{C}-182^{\circ} \mathrm{C} . \mathbf{I R}(\mathrm{KBr}): 1580,1597$, 1655, 3378. ${ }^{1} \mathrm{H}$ NMR $\left(\mathrm{CD}_{3} \mathrm{OD}\right) \delta: 4.45\left(\mathrm{~s}, \mathrm{CH}_{2}\right) ; 7.26$ - 7.38 (m, 5H). Anal.Calcd.for $\mathrm{C}_{8} \mathrm{H}_{10} \mathrm{~N}_{4} \mathrm{O}_{2}+0.13 \mathrm{H}_{2} \mathrm{O}: \mathrm{C}$, 48.89; H, 5.26; N, 28.51. Found: C, 48.80; H, 4.95; N, 28.61.

1-nitro-3-(2-(trifluoromethyl)benzyl)guanidine(9):

The title compound was prepared according to the above procedure using 2-(trifluoromethyl)benzylamine (0.48 mL, $3.10 \mathrm{mmol})$ and 1-methyl-3-nitro-1-nitrosoguanidine (455 $\mathrm{mg}, 3.44 \mathrm{mmol}$ ) in a mixture of ethanol and water (50/50, v/v, $14 \mathrm{~mL})$. The reaction afforded $(711 \mathrm{mg}, 86 \%)$ of $\mathbf{9}$ as a white solid. $\mathrm{mp}=153^{\circ} \mathrm{C}-154^{\circ} \mathrm{C}$. IR (KBr): 1535, 1604, 1649, 3182, 3314, 3409. ${ }^{1} \mathbf{H}$ NMR $\left(\mathrm{CD}_{3} \mathrm{OD}\right) \delta: 4.65\left(\mathrm{~s}, \mathrm{CH}_{2}\right) ; 7.46-7.74(\mathrm{~m},=\mathrm{CH})$.

Anal.Calcd.for $\mathrm{C}_{9} \mathrm{H}_{9} \mathrm{~F}_{3} \mathrm{~N}_{4} \mathrm{O}_{2}$ : C, 41.23; H, 3.46; N, 21.37. Found: C, 41.40; H, 3.39; N, 21.34.

1-nitro-3-(3-(trifluoromethyl)benzyl)guanidine (10):

The title compound was prepared according to the above procedure using 3-(trifluoromethyl)benzylamine $(0.50 \mathrm{~mL}, 3.49 \mathrm{mmol})$ and 1-methyl-3-nitro-1-nitrosoguanidine $(510 \mathrm{mg}, 3.47 \mathrm{mmol})$ in a mixture of ethanol and water $(50 / 50, \mathrm{v} / \mathrm{v}, 16 \mathrm{~mL})$. The reaction afforded $(794 \mathrm{mg}, 88 \%)$ of 10 as a white solid. $\mathrm{mp}=164.5^{\circ} \mathrm{C}-$ $165.5^{\circ} \mathrm{C}$. IR (KBr): 1617, 1649, 3157, 3487, 3569. ${ }^{1} \mathbf{H}$ NMR $\left(\mathrm{CD}_{3} \mathrm{OD}\right) \delta: 4.54\left(\mathrm{~s}, \mathrm{CH}_{2}\right) ; 7.52-7.64(\mathrm{~m},=\mathrm{CH})$. Anal.Calcd.for $\mathrm{C}_{9} \mathrm{H}_{9} \mathrm{~F}_{3} \mathrm{~N}_{4} \mathrm{O}_{2}$ : C, 41.23; H, 3.46; N, 21.37. Found: C, 41.18; H, 3.26; N, 21.23.

1-nitro-3-(4-(trifluoromethyl)benzyl)guanidine (11):

The title compound was prepared according to the above procedure using 4-(trifluoromethyl)benzylamine (0.45 mL, $3.16 \mathrm{mmol}$ ) and 1-methyl-3-nitro-1-nitrosoguanidine (471 mg, $3.20 \mathrm{mmol}$ ) in a mixture of ethanol and water (50/50, v/v, $12 \mathrm{~mL})$. The reaction afforded $(713 \mathrm{mg}, 85 \%)$ of 11 as a white solid. $\mathrm{mp}=162.5^{\circ} \mathrm{C}-$ $163.5^{\circ} \mathrm{C}$. IR (KBr): 1594, 1655, 3215, 3304, 3385. ${ }^{1} \mathbf{H}$ NMR $\left(\mathrm{CD}_{3} \mathrm{OD}\right) \delta: 4.54\left(\mathrm{~s}, \mathrm{CH}_{2}\right) ; 7.50\left(\mathrm{~d},{ }^{3} \mathrm{~J}=4.0 \mathrm{~Hz}\right.$ $=\mathrm{CH}) ; 7.65\left(\mathrm{~d},{ }^{3} \mathrm{~J}=4.1 \mathrm{~Hz},=\mathrm{CH}\right)$. Anal.Calcd.for $\mathrm{C}_{9} \mathrm{H}_{9} \mathrm{~F}_{3} \mathrm{~N}_{4} \mathrm{O}_{2}$ : C, 41.23; H, 3.46; N, 21.37. Found: C, 41.07; $\mathrm{H}$, 3.55; N, 21.35.

1-(2-amino-6-fluorobenzyl)-3-nitroguanidine(12):

The title compound was prepared according to the above procedure using 2-fluoro-6-amino-benzylamine (352 $\mathrm{mg}, 2.14 \mathrm{mmol}$ ) and 1-methyl-3-nitro-1-nitrosoguanidine (336 mg, $2.29 \mathrm{mmol}$ ) in a mixture of ethanol and water (50/50, v/v, $10 \mathrm{~mL})$. The reaction afforded (345 mg, 67\%) of 12 as a white solid. $\mathrm{mp}=176^{\circ} \mathrm{C}$, decomposition. IR (KBr): 1556, 1607, 3305, 3369. ${ }^{1} \mathbf{H}$ NMR (CD $\left.{ }_{3} \mathrm{OD}\right) \delta: 4.37\left(\mathrm{~d},{ }^{4} \mathrm{~J}_{\mathrm{H}, \mathrm{F}}=3.7 \mathrm{~Hz}, \mathrm{CH}_{2}\right) ; 6.42\left(\mathrm{t},{ }^{3} \mathrm{~J}=9.0 \mathrm{~Hz}\right.$, 
$=\mathrm{CH}) ; 6.56\left(\mathrm{~d},{ }^{3} \mathrm{~J}=8.1 \mathrm{~Hz},=\mathrm{CH}\right) ; 6.56\left(\mathrm{dd},{ }^{3} \mathrm{~J}_{\mathrm{H}, \mathrm{F}}=14.9 \mathrm{~Hz},{ }^{3} \mathrm{~J}=8.0 \mathrm{~Hz},=\mathrm{CH}\right)$. Anal.Calcd.for $\mathrm{C}_{8} \mathrm{H}_{10} \mathrm{FN}_{5} \mathrm{O}_{2}+$ $0.15 \mathrm{H}_{2} \mathrm{O}$ : C, 41.79; H, 4.52; N, 30.46. Found: C, 41.91; H, 4.25; N, 29.93.

1-nitro-3-(thiophen-2-ylmethyl)guanidine(13):

The title compound was prepared according to the above procedure using 2-aminomethyl-thiophene $(0.36 \mathrm{~mL}$, $3.50 \mathrm{mmol})$ and 1-methyl-3-nitro-1-nitrosoguanidine (515 $\mathrm{mg}, 3.50 \mathrm{mmol})$ in a mixture of ethanol and water (50/50, v/v, $16 \mathrm{~mL}$ ). The reaction afforded (577 $\mathrm{mg}, 84 \%)$ of $\mathbf{1 3}$ as a white solid. $\mathrm{mp}=147^{\circ} \mathrm{C}-147.5^{\circ} \mathrm{C}$. IR (KBr): 1541, 1597, 1650, 3167, 3318, 3378. ${ }^{1} \mathbf{H}$ NMR $\left(\mathrm{CD}_{3} \mathrm{OD}\right) \delta: 4.62\left(\mathrm{~s}, \mathrm{CH}_{2}\right) ; 6.96\left(\mathrm{dd},{ }^{3} \mathrm{~J}=4.9 \mathrm{~Hz},{ }^{3} \mathrm{~J}=3.8\right.$ $\mathrm{Hz},-\mathrm{CH}=) ; 7.04(\mathrm{~m},-\mathrm{CH}=) ; 7.33\left(\mathrm{~d},{ }^{3} \mathrm{~J}=4.9 \mathrm{~Hz}, \mathrm{~S}-\mathrm{CH}=\right)$. Anal.Calcd.for $\mathrm{C}_{6} \mathrm{H}_{8} \mathrm{~N}_{4} \mathrm{O}_{2} \mathrm{~S}: \mathrm{C}, 35.99 ; \mathrm{H}, 4.03 ; \mathrm{N}$, 27.98. Found: C, 35.94; H, 4.01; N, 28.05.

1-nitro-3-(2-(thiophen-2-yl)ethyl)guanidine(14):

The title compound was prepared according to the above procedure using 2-(2-aminoethyl)-thiophene (984 $\mathrm{mg}, 7.73 \mathrm{mmol})$ and 1-methyl-3-nitro-1-nitrosoguanidine $(1.144 \mathrm{~g}, 7.73 \mathrm{mmol})$ in a mixture of ethanol and water $(50 / 50, \mathrm{v} / \mathrm{v}, 20 \mathrm{~mL})$. The reaction afforded $(1.467 \mathrm{~g}, 91 \%)$ of 14 as a white solid. $\mathrm{mp}=137^{\circ} \mathrm{C}-137.5^{\circ} \mathrm{C}$. IR (KBr): 1553, 1594, 1650, 3181, 3302, 3381. ${ }^{1} \mathbf{H}$ NMR (CD $\left.{ }_{3} \mathrm{OD}\right) \delta: 3.11\left(\mathrm{t},{ }^{3} \mathrm{~J}=6.4 \mathrm{~Hz}, \mathrm{CH}_{2 \beta}\right) ; 3.51\left(\mathrm{t},{ }^{3} \mathrm{~J}=6.9\right.$ $\mathrm{Hz}, \mathrm{CH}_{2 \alpha}$ ); 6.91-6.95 (m, - $\left.\mathrm{CH}=\right) ; 7.23$ (dd, $\left.{ }^{3} \mathrm{~J}=5.0 \mathrm{~Hz},{ }^{5} \mathrm{~J}=1.1 \mathrm{~Hz}, \mathrm{~S}-\mathrm{CH}=\right)$. Anal.Calcd.for $\mathrm{C}_{7} \mathrm{H}_{10} \mathrm{~N}_{4} \mathrm{O}_{2} \mathrm{~S}: \mathrm{C}$ 39.24; H, 4.70; N, 26.15. Found: C, 39.26; H, 4.50; N, 26.02.

1-nitro-3-(pyridin-3-yl)guanidine(15):

3-amino-pyridine (330 mg, $3.51 \mathrm{mmol}$ ) was added to a suspension of 1-methyl-3-nitro-1-nitrosoguanidine (516 mg, $3.51 \mathrm{mmol}$ ) in a mixture of ethanol and water (50/50, v/v, $10 \mathrm{~mL}$ ). The mixture was reacted overnight at $90^{\circ} \mathrm{C}-95^{\circ} \mathrm{C}$. The reaction mixture was dry evaporated and the crude product purified by flash chromatography (silica gel, $\mathrm{CHCl}_{3}(95 \%) / \mathrm{MeOH}(5 \%)$ ). The expected compound $\mathbf{1 5}$ was isolated as a white solid (91 mg, $14 \%) . \mathrm{mp}=194.5^{\circ} \mathrm{C}-195.5^{\circ} \mathrm{C} . \mathbf{I R}(\mathrm{KBr}): 1555,1588,1636,3074,3219,3328 .{ }^{1} \mathbf{H}$ NMR $\left(\mathrm{CD}_{3} \mathrm{OD}\right) \delta: 7.46(\mathrm{dd}$, $\mathrm{J}_{1}=8.1 \mathrm{~Hz}, \mathrm{~J}_{2}=5.0 \mathrm{~Hz}, \mathrm{H}_{5}$ ); 7.90 (ddd, $\left.\mathrm{J}_{1}=8.2 \mathrm{~Hz}, \mathrm{~J}_{3}=2.5 \mathrm{~Hz}, \mathrm{~J}_{4}=1.4 \mathrm{~Hz}, \mathrm{H}_{4}\right) ; 8.37$ (dd, $\mathrm{J}_{2}=4.8 \mathrm{~Hz}, \mathrm{~J}_{4}=1.3$ $\mathrm{Hz}, \mathrm{H}_{6}$ ); 8.52 (d, $\mathrm{J}_{3}=2.2 \mathrm{~Hz}, \mathrm{H}_{2}$ ). Anal.Calcd.for $\mathrm{C}_{6} \mathrm{H}_{7} \mathrm{~N}_{5} \mathrm{O}_{2}$ : C, 39.78; $\mathrm{H}, 3.89 ; \mathrm{N}, 38.66$. Found: $\mathrm{C}, 39.35 ; \mathrm{H}$, $3.71 ; \mathrm{N}, 38.81$.

1-nitro-3-(pyridin-2-yl)guanidine(16):

2-amino-pyridine (361 $\mathrm{mg}, 3.84 \mathrm{mmol}$ ) was added to a suspension of 1-methyl-3-nitro-1-nitrosoguanidine (514 mg, $3.50 \mathrm{mmol}$ ) in a mixture of ethanol and water $(50 / 50, \mathrm{v} / \mathrm{v}, 10 \mathrm{~mL})$. The mixture was reacted $2 \mathrm{~h}$ at $60^{\circ} \mathrm{C}$ and overnight at $90^{\circ} \mathrm{C}$. The reaction mixture was dry evaporated and the crude product purified by flash chromatography (silica gel, $\mathrm{CHCl}_{3}(90 \%) / \mathrm{MeOH}(10 \%)$ ). The expected compound $\mathbf{1 6}$ was isolated as a yellowish solid (45 mg, 7\%). It was further purified be crystallization from $\mathrm{MeOH}$ to afford a white solid. $\mathrm{mp}=$ $226^{\circ} \mathrm{C}-227^{\circ} \mathrm{C}$. IR (KBr): $1543,1559,1600,1610,3160$ - 3600. ${ }^{1} \mathbf{H}$ NMR $\left(\mathrm{CD}_{3} \mathrm{OD}\right) \delta: 7.01\left(\mathrm{~d}, \mathrm{~J}_{1}=8.4 \mathrm{~Hz}, \mathrm{H}_{3}\right)$; 7.08 (ddd, $\mathrm{J}_{2}=7.5 \mathrm{~Hz}, \mathrm{~J}_{3}=7.1 \mathrm{~Hz}, \mathrm{~J}_{4}=0.7 \mathrm{~Hz}, \mathrm{H}_{5}$ ); 7.74 (ddd, $\mathrm{J}_{1}=9.1 \mathrm{~Hz}, \mathrm{~J}_{2}=7.4 \mathrm{~Hz}, \mathrm{~J}_{5}=1.8 \mathrm{~Hz}, \mathrm{H}_{4}$ ); 8.28 (ddd, $\mathrm{J}_{3}=5.1 \mathrm{~Hz}, \mathrm{~J}_{5}=1.7 \mathrm{~Hz}, \mathrm{~J}_{6}=0.7 \mathrm{~Hz}, \mathrm{H}_{6}$ ). Anal.Calcd.for $\mathrm{C}_{6} \mathrm{H}_{7} \mathrm{~N}_{5} \mathrm{O}_{2}+0.25 \mathrm{H}_{2} \mathrm{O}: \mathrm{C}, 38.82 ; \mathrm{H}, 4.07 ; \mathrm{N}$, 37.72. Found: C, 38.88; H, 3.85; N, 37.51.

1-nitro-3-(pyridin-3-ylmethyl)guanidine(17):

The title compound was prepared according to the above procedure (compound 5) using 2-aminomethyl-pyridine $(0.35 \mathrm{~mL}, 3.40 \mathrm{mmol})$ and 1-methyl-3-nitro-1-nitrosoguanidine $(500 \mathrm{mg}, 3.40 \mathrm{mmol})$ in a mixture of ethanol and water (50/50, v/v, $9 \mathrm{~mL}$ ). The reaction afforded (607 mg, 92\%) of $\mathbf{1 7}$ as a white solid. 17 (607 mg, 92\%). It was purified by crystallization from $\mathrm{MeOH} . \mathrm{mp}=180^{\circ} \mathrm{C}-181^{\circ} \mathrm{C}$. IR $(\mathrm{KBr}): 1542,1568,1606,1656$, 3155, 3304, 3382. ${ }^{1} \mathbf{H}$ NMR $\left(\mathrm{CD}_{3} \mathrm{OD}\right) \delta: 4.56\left(\mathrm{~s}, \mathrm{CH}_{2}\right) ; 7.33\left(\mathrm{dd}, \mathrm{J}_{1}=6.9 \mathrm{~Hz}, \mathrm{~J}_{2}=5.3 \mathrm{~Hz}, \mathrm{H}_{5}\right) ; 7.41\left(\mathrm{~d}, \mathrm{~J}_{3}=7.8\right.$ $\mathrm{Hz}, \mathrm{H}_{3}$ ); 7.83 (ddd, $\left.\mathrm{J}_{1} \sim \mathrm{J}_{3} \sim 7.7 \mathrm{~Hz}, \mathrm{~J}_{4}=1.5 \mathrm{~Hz}, \mathrm{H}_{4}\right) ; 8.53\left(\mathrm{~d}, \mathrm{~J}_{2}=4.2 \mathrm{~Hz}, \mathrm{H}_{6}\right.$ ). Anal.Calcd.for $\mathrm{C}_{7} \mathrm{H}_{9} \mathrm{~N}_{5} \mathrm{O}_{2}: \mathrm{C}$, 43.08; H, 4.65; N, 35.88. Found: C, 43.03; H, 4.70; N, 35.93.

1-nitro-3-(2-(pyridin-3-yl)ethyl)guanidine(18):

The title compound was prepared according to the above procedure (compound 5) using 2-(2-aminoethyl)pyridine $(0.41 \mathrm{~mL}, 3.45 \mathrm{mmol})$ and 1-methyl-3-nitro-1-nitrosoguanidine $(520 \mathrm{mg}, 3.43 \mathrm{mmol})$ in a mixture of ethanol and water (50/50, v/v, $13 \mathrm{~mL}$ ). The reaction afforded $(619 \mathrm{mg}, 92 \%)$ of $\mathbf{1 8}$ as a brown solid. It was purified by crystallization from a mixture of $\mathrm{CHCl}_{3}$ and $\mathrm{MeOH}$ to afford a white yellowish solid. $\mathrm{mp}=142^{\circ} \mathrm{C}$ $142.5^{\circ} \mathrm{C}$. IR (KBr): $1570,1592,1612,1632,3117,3186,3245,3342 .{ }^{1} \mathbf{H}$ NMR $\left(\mathrm{CD}_{3} \mathrm{OD}\right) \delta: 3.06\left(\mathrm{t},{ }^{3} \mathrm{~J}=6.8 \mathrm{~Hz}\right.$, $\left.\mathrm{CH}_{2 \beta}\right) ; 3.61\left(\mathrm{t},{ }^{3} \mathrm{~J}=7.0 \mathrm{~Hz}, \mathrm{CH}_{2 \alpha}\right.$ ); $7.28\left(\mathrm{dd}, \mathrm{J}_{1}=7.1 \mathrm{~Hz}, \mathrm{~J}_{2}=5.2 \mathrm{~Hz}, \mathrm{H}_{5}\right) ; 7.35\left(\mathrm{~d}, \mathrm{~J}_{3}=7.8 \mathrm{~Hz}, \mathrm{H}_{3}\right) ; 7.77$ (ddd, $\mathrm{J}_{1}$ $\left.\sim \mathrm{J}_{3} \sim 7.7 \mathrm{~Hz}, \mathrm{~J}_{4}=1.7 \mathrm{~Hz}, \mathrm{H}_{4}\right) ; 8.49\left(\mathrm{~d}, \mathrm{~J}_{2}=4.4 \mathrm{~Hz}, \mathrm{H}_{6}\right)$. Anal.Calcd.for $\mathrm{C}_{8} \mathrm{H}_{11} \mathrm{~N}_{5} \mathrm{O}_{2}$ : C, 45.93; H, 5.30; N, 33.48 . Found: C, 45.95; H, 5.37; N, 33.29.

$\mathrm{N}$-nitropiperidine-1-carboximidamide(19): 
The title compound was prepared according to the above procedure (compound 5 ) using piperidine (0.35 $\mathrm{mL}$, $3.54 \mathrm{mmol}$ ) and 1-methyl-3-nitro-1-nitrosoguanidine (510 g, $3.47 \mathrm{mmol})$ in a mixture of ethanol and water (50/50, v/v, $14 \mathrm{~mL}$ ). The reaction afforded (340 mg, 57\%) of 19 as a white solid. $\mathrm{mp}=151^{\circ} \mathrm{C}-153^{\circ} \mathrm{C}$. IR $(\mathrm{KBr})$ : 1562, 1615, 3210, 3263, 3362. ${ }^{1} \mathrm{H}$ NMR (CD $\left.\mathrm{OD}\right) \delta: 1.58-1.73\left(\mathrm{~m}, 6 \mathrm{H}, \mathrm{CH}_{2}\right) ; 3.54-3.57\left(\mathrm{~m}, 4 \mathrm{H}, \mathrm{CH}_{2}\right)$. Anal.Calcd.for $\mathrm{C}_{6} \mathrm{H}_{12} \mathrm{~N}_{4} \mathrm{O}_{2}$ : C, 41.85; H, 7.02; N, 32.54. Found: C, 41.95; H, 7.05; N, 32.48 .

1-nitro-3-(piperidin-1-yl)guanidine(20):

The title compound was prepared according to the above procedure (compound 5) using 1-aminopiperidine (0.34 mL, $3.15 \mathrm{mmol}$ ) and 1-methyl-3-nitro-1-nitrosoguanidine (460 g, $3.13 \mathrm{mmol}$ ) in a mixture of ethanol and water (50/50, v/v, $14 \mathrm{~mL})$. The reaction afforded (216 mg, $37 \%)$ of 20 as a white solid. $\mathrm{mp}=180^{\circ} \mathrm{C}-181^{\circ} \mathrm{C}$. IR (KBr): 1565, 1605, 3210, 3301, 3439. ${ }^{1} \mathbf{H}$ NMR (CD $\mathrm{CD}_{3} \mathrm{O} \delta: 1.19$ - 1.23 (m, 1H, C(H)H); 1.70 - 1.75 (m, $5 \mathrm{H}$, $\left.\mathrm{CH}_{2}\right)$; 2.50-2.56 (m, 2H, $\left.\mathrm{CH}_{2}\right)$; 2.93-2.99 (m, 2H, $\mathrm{CH}_{2}$ ). Anal.Calcd.for $\mathrm{C}_{6} \mathrm{H}_{13} \mathrm{~N}_{5} \mathrm{O}_{2}$ : C, 38.49; H, 6.95; N, 37.41. Found: C, 38.29; H, 6.77; N, 37.25.

1-nitro-3-(2-(piperidin-1-yl)ethyl)guanidine(21):

The title compound was prepared according to the above procedure (compound 5) using 1-(2-aminoethyl)piperidine (0.515 mL, $3.61 \mathrm{mmol})$, 1-methyl-3-nitro-1-nitrosoguanidine (529 $\mathrm{mg}, 3.59 \mathrm{mmol}$ ) in a mixture of ethanol and water (50/50, v/v, $9 \mathrm{~mL})$ affording $(634 \mathrm{mg}, 81 \%)$ of 21 as a white solid. $\mathrm{mp}=168^{\circ} \mathrm{C}-168.5^{\circ} \mathrm{C}$. IR (KBr): 1543, 1586, 1641, 3119, 3239, 3367. ${ }^{1} \mathbf{H}$ NMR (CD $\left.3 \mathrm{OD}\right) \delta: 1.46-1.49$ (m, $\left.\mathrm{CH}_{2 \gamma}\right) ; 1.57$ - $1.64\left(\mathrm{~m}, \mathrm{CH}_{2 \beta}\right)$; 2.48/2.252 - 2.56 (s and m, $\mathrm{CH}_{2 \alpha / \alpha^{\prime}}$ ); 3.35 (t, ${ }^{3} \mathrm{~J}=5.9 \mathrm{~Hz}, \mathrm{CH}_{2 \beta}$ ). Anal.Calcd.for $\mathrm{C}_{8} \mathrm{H}_{17} \mathrm{~N}_{5} \mathrm{O}_{2}$ : C, 44.64; H, 7.96; N, 32.54. Found: C, 44.28; H, 7.82; N, 32.13.

N-nitro-3,4-dihydroisoquinoline-2(1H)-carboximidamide (22):

The title compound was prepared according to the above procedure (compound 5) using 2-isoquinoline (0.39 $\mathrm{mL}, 3.12 \mathrm{mmol}$ ), 1-methyl-3-nitro-1-nitrosoguanidine (466 mg, $3.17 \mathrm{mmol}$ ) in a mixture of ethanol and water (50/50, v/v, $14 \mathrm{~mL}$ ) affording (587 mg, 89\%) of 22 as a white solid. $\mathrm{mp}=119.5^{\circ} \mathrm{C}-121^{\circ} \mathrm{C}$. IR $(\mathrm{KBr}): 1571$, 1610, 3260, 3386. ${ }^{1} \mathbf{H}$ NMR $\left(\mathrm{CD}_{3} \mathrm{OD}\right) \delta: 2.94\left(\mathrm{t},{ }^{3} \mathrm{~J}=6.0 \mathrm{~Hz}, \mathrm{CH}_{2}\right) ; 3.74\left(\mathrm{t},{ }^{3} \mathrm{~J}=6.0 \mathrm{~Hz}, \mathrm{CH}_{2}\right) ; 6.68\left(\mathrm{~s}, \mathrm{CH}_{2}\right) ; 7.16$ - 7.23 (m, 4H, -CH=). Anal.Calcd.for $\mathrm{C}_{10} \mathrm{H}_{12} \mathrm{~N}_{4} \mathrm{O}_{2}$ : C, 54.54; H, 5.49; N, 25.44. Found: C, 54.55; H, 5.37; N, 25.52.

N-nitromorpholine-4-carboximidamide(23):

The title compound was prepared according to the above procedure (compound 5$)$ using morpholine $(0.28 \mathrm{~mL}$, $3.20 \mathrm{mmol}$ ), 1-methyl-3-nitro-1-nitrosoguanidine (466 mg, $3.15 \mathrm{mmol}$ ) in a mixture of ethanol and water (50/50, v/v, $8 \mathrm{~mL}$ ) affording (404 mg, 78\%) of 23 as a white solid. $\mathrm{mp}=186.5^{\circ} \mathrm{C}-187^{\circ} \mathrm{C}$. IR (KBr): 1560, 1612, 3289, 3401. ${ }^{1} \mathbf{H}$ NMR ( $\left.\mathrm{CD}_{3} \mathrm{OD}\right) \delta$ : 3.57 (t, $\left.{ }^{3} \mathrm{~J}=4.9 \mathrm{~Hz}, \mathrm{~N}-\mathrm{CH}_{2}\right)$; $3.69\left(\mathrm{t},{ }^{3} \mathrm{~J}=4.9 \mathrm{~Hz}, \mathrm{O}-\mathrm{CH}_{2}\right)$. Anal.Calcd.for $\mathrm{C}_{5} \mathrm{H}_{10} \mathrm{~N}_{4} \mathrm{O}_{3}$ : C, 34.48; H, 5.79; N, 32.17. Found: C, 34.39; H, 5.44; N, 32.46.

1-morpholino-3-nitroguanidine(24):

The title compound was prepared according to the above procedure (compound 5) using 1-aminomorpholine (0.32 mL, $3.32 \mathrm{mmol}$ ) and 1-methyl-3-nitro-1-nitrosoguanidine (492 g, $3.34 \mathrm{mmol}$ ) in a mixture of ethanol and water (50/50, v/v, $8 \mathrm{~mL})$. The reaction afforded $(420 \mathrm{mg}, 68 \%)$ of 24 as a white solid. $\mathrm{mp}=243^{\circ} \mathrm{C}$, decomposition. IR (KBr): 1582, 1621, 3215, 3267, 3418. ${ }^{1} \mathbf{H}$ NMR (CD $\left.\mathrm{CD}_{3} \mathrm{O}\right) \delta: 2.75$ - 2.90 (m, 4H, OCH $)$; 3.30 - 3.65 (m, $4 \mathrm{H}, \mathrm{NCH}_{2}$ ). Anal.Calcd.for $\mathrm{C}_{5} \mathrm{H}_{11} \mathrm{~N}_{5} \mathrm{O}_{3}$ : C, 31.75; H, 5.86; N, 37.02. Found: C, 32.14; H, 5.62; N, 36.89.

3-morpholino-N-nitropropanimidamide(25):

The title compound was prepared according to the above procedure (compound 5) using 4-(2-aminoethyl)morpholine $(0.49 \mathrm{~mL}, 3.73 \mathrm{mmol})$, 1-methyl-3-nitro-1-nitrosoguanidine (543 mg, $3.69 \mathrm{mmol}$ ) in a mixture of ethanol and water (50/50, v/v, $9 \mathrm{~mL}$ ) affording (633 mg, 79\%) of 25 as a white solid. $\mathrm{mp}=190.5^{\circ} \mathrm{C}-191.5^{\circ} \mathrm{C}$. IR (KBr): 1581, 1655, 3114, 3236, 3367. ${ }^{1} \mathbf{H}$ NMR $\left(\mathrm{CD}_{3} \mathrm{OD}\right) \delta$ : 2.51/2.58 (s and s, $\mathrm{CH}_{2 \alpha / \alpha}$ ); 3.37 (t, ${ }^{3} \mathrm{~J}=5.9 \mathrm{~Hz}$, $\mathrm{CH}_{2 \beta}$ ); $3.70\left(\mathrm{t},{ }^{3} \mathrm{~J}=4.6 \mathrm{~Hz}, \mathrm{CH}_{2 \beta}\right)$. Anal.Calcd.for $\mathrm{C}_{7} \mathrm{H}_{15} \mathrm{~N}_{5} \mathrm{O}_{3}: \mathrm{C}, 38.70 ; \mathrm{H}, 6.96 ; \mathrm{N}, 32.24$. Found: C, 38.74; $\mathrm{H}$, $6.82 ; \mathrm{N}, 32.17$.

1-nitro-3-((tetrahydrothiophen-2-yl)methyl)guanidine(26):

The title compound was prepared according to the above procedure (compound 5) using tetrahydrofurfurylamine (0.36 mL, $3.49 \mathrm{mmol})$, 1-methyl-3-nitro-1-nitrosoguanidine (510 mg, $3.47 \mathrm{mmol}$ ) in a mixture of ethanol and water (50/50, v/v, $9 \mathrm{~mL}$ ) affording (527 mg, 79\%) of 26 as a white solid. $\mathrm{mp}=84^{\circ} \mathrm{C}-85^{\circ} \mathrm{C}$. IR (KBr): 1603 ,

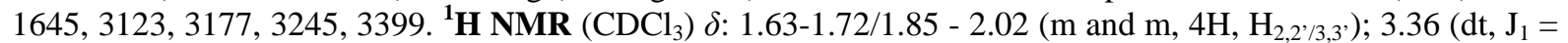
$\left.15.1 \mathrm{~Hz}, \mathrm{~J}_{2}=5.9 \mathrm{~Hz}, \mathrm{H}_{4}\right)$; 3.61 (ddd, $\mathrm{J}_{1}=15.1 \mathrm{~Hz}, \mathrm{~J}_{3}=3.2 \mathrm{~Hz}, \mathrm{~J}_{4}=1.65 \mathrm{~Hz}, \mathrm{H}_{4}$ ); $3.75\left(\mathrm{dd}, \mathrm{J}_{5}=13.4 \mathrm{~Hz}, \mathrm{~J}_{6}=6.8\right.$ $\mathrm{Hz}, \mathrm{CH}-\mathrm{NH}$ ); 3.86 (dd, $\left.\mathrm{J}_{5}=14.9 \mathrm{~Hz}, \mathrm{~J}_{7}=6.8 \mathrm{~Hz}, \mathrm{CH}-\mathrm{NH}\right) ; 4.00$ - 4.08 (m, $\mathrm{H}_{1}$ ). Anal.Calcd.for $\mathrm{C}_{6} \mathrm{H}_{12} \mathrm{~N}_{4} \mathrm{O}_{3}$ : C, 38.30; H, 6.43; N, 29.77. Found: C, 38.05; H, 6.40; N, 29.54. 
1-nitro-3-(thiophen-2-ylmethyl)guanidine(27):

The title compound was prepared according to the above procedure (compound 5) using furfurylamine $(0.31$ $\mathrm{mL}, 3.51 \mathrm{mmol})$, 1-methyl-3-nitro-1-nitrosoguanidine (500 $\mathrm{mg}, 3.40 \mathrm{mmol})$ in a mixture of ethanol and water (50/50, v/v, $9 \mathrm{~mL}$ ) affording (388 $\mathrm{mg}, 62 \%)$ of 27 as a white solid. $\mathrm{mp}=138.5^{\circ} \mathrm{C}-140^{\circ} \mathrm{C}$. IR $(\mathrm{KBr}): 1544,1594$, 1652, 3175, 3322, 3387. ${ }^{1} \mathrm{H}$ NMR $\left(\mathrm{CD}_{3} \mathrm{OD}\right) \delta: 4.36\left(\mathrm{~s}, 2 \mathrm{H}, \mathrm{CH}_{2}\right) ; 6.33-6.37(\mathrm{~m}, 2 \mathrm{H},-\mathrm{CH}=) ; 7.46(\mathrm{~s}, 1 \mathrm{H}$, - $\mathrm{CH}=$ ). Anal.Calcd.for $\mathrm{C}_{6} \mathrm{H}_{8} \mathrm{~N}_{4} \mathrm{O}_{3}$ : C, 39.13; H, 4.38; N, 30.42. Found: C, 39.04; H, 4.32; N, 30.33.

1-((5-methylthiophen-2-yl)methyl)-3-nitroguanidine(28):

The title compound was prepared according to the above procedure (compound 5) using 5-methylfurfurylamine (0.44 mL, $3.95 \mathrm{mmol})$, 1-methyl-3-nitro-1-nitrosoguanidine (573 $\mathrm{mg}, 3.90 \mathrm{mmol}$ ) in a mixture of ethanol and water (50/50, v/v, $9 \mathrm{~mL}$ ) affording $(700 \mathrm{mg}, 91 \%)$ of 28 as an off white solid. $\mathrm{mp}=162.5^{\circ} \mathrm{C}-163^{\circ} \mathrm{C}$. IR (KBr): 1549, 1602, 1651, 3161, 3306, 3381. ${ }^{1} \mathbf{H}$ NMR $\left(\mathrm{CD}_{3} \mathrm{OD}\right) \delta: 2.25\left(\mathrm{~s}, \mathrm{CH}_{3}\right) ; 4.37\left(\mathrm{~s}, \mathrm{CH}_{2}\right) ; 5.94\left(\mathrm{~d},{ }^{3} \mathrm{~J}=2.1\right.$ $\mathrm{Hz}, \mathrm{CH}) ; 6.20\left(\mathrm{~d},{ }^{3} \mathrm{~J}=2.6 \mathrm{~Hz}, \mathrm{CH}\right)$. Anal.Calcd.for $\mathrm{C}_{7} \mathrm{H}_{10} \mathrm{~N}_{4} \mathrm{O}_{3}$ : C, 42.42; H, 5.09; N, 28.27. Found: C, 42.45; $\mathrm{H}$, 4.92; N, 28.05.

N-nitropyrrolidine-1-carboximidamide(29):

The title compound was prepared according to the above procedure (compound 5$)$ using pyrolidine $(0.30 \mathrm{~mL}$, $3.59 \mathrm{mmol})$ and 1-methyl-3-nitro-1-nitrosoguanidine $(520 \mathrm{~g}, 3.54 \mathrm{mmol})$ in a mixture of ethanol and water (50/50, v/v, $12 \mathrm{~mL})$. The reaction afforded $(375 \mathrm{mg}, 67 \%)$ of 29 as a white solid. $\mathrm{mp}=188^{\circ} \mathrm{C}-189^{\circ} \mathrm{C}$. IR $(\mathrm{KBr})$ : 1563, 1617, 3218, 3279, 3431. ${ }^{1} \mathbf{H}$ NMR $\left(\mathrm{CD}_{3} \mathrm{OD}\right) \delta: 1.94-2.00\left(\mathrm{~m}, 4 \mathrm{H}, \mathrm{CH}_{2}\right)$; 3.41-3.47 (m, 4H, $\left.\mathrm{CH}_{2}\right)$. Anal.Calcd.for $\mathrm{C}_{5} \mathrm{H}_{10} \mathrm{~N}_{4} \mathrm{O}_{2}$ : C, 37.97; H, 6.37; N, 35.42. Found: C, 38.10; H, 6.44; N, 35.63.

1-nitro-3-(2-(pyrrolidin-1-yl)ethyl)guanidine(30)

The title compound was prepared according to the above procedure (compound 5) using 1-(2-aminoethyl)pyrrolidine $(0.48 \mathrm{~mL}, 3.79 \mathrm{mmol}), 1$-methyl-3-nitro-1-nitrosoguanidine ( $549 \mathrm{mg}, 3.73 \mathrm{mmol}$ ) in absolute ethanol $(6 \mathrm{~mL})$ affording, after cooling at $-10^{\circ} \mathrm{C},(250 \mathrm{mg}, 33 \%)$ of 30 as a white solid. $\mathrm{mp}=119^{\circ} \mathrm{C}-120^{\circ} \mathrm{C}$. IR (KBr): 1544, 1593, 1640, 3119, 3236, 3361. ${ }^{1} \mathbf{H}$ NMR $\left(\mathrm{CD}_{3} \mathrm{OD}\right) \delta: 1.79-1.83$ (m, $\left.\mathrm{CH}_{2 \beta}\right) ; 2.60 / 2.71$ (s and s, $\mathrm{CH}_{2 \alpha / \alpha^{\prime}}$ ); $3.78\left(\mathrm{t},{ }^{3} \mathrm{~J}=6.2 \mathrm{~Hz}, \mathrm{CH}_{2 \beta}\right.$ ). Anal.Calcd.for $\mathrm{C}_{7} \mathrm{H}_{15} \mathrm{~N}_{5} \mathrm{O}_{2}$ : C, 41.78; H, 7.51; N, 34.80. Found: C, 41.90; H, 7.46; N, 34.74.

\subsection{Biology}

Assay for Nitric Oxide Synthase activity: Compounds were assayed for nitric oxide synthase activity using a citrulline formation assay with affinity purified enzymes and L-[2,3- $\left.{ }^{3} \mathrm{H}\right]$ arginine as the substrate [23]. Enzymes were prepared as previously described [24]. For enzyme assays, iNOS, eNOS or nNOS was incubated in $150 \mu \mathrm{L}$

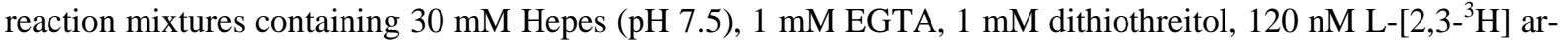
ginine (New England Nuclear, final concentration 200,000 dpm/reaction mix), $100 \mu \mathrm{M}$ NADPH, and $300 \mu \mathrm{M}$ tetrahydrobiopterin. For assays with nNOS and eNOS, $6 \mu \mathrm{M}$ calmodulin and $0.85 \mathrm{mM} \mathrm{Ca}^{2+}$ were also added. Reactions, run in duplicate in $5 \mathrm{ml}$ glass scintillation vials, were initiated by the addition of iNOS, eNOS or nNOS with and without increasing concentrations of the inhibitors. After $30 \mathrm{~min}$ at $30^{\circ} \mathrm{C}$, reactions were stopped by the addition of $1 \mathrm{ml}$ of AF 50WX8 resin in $20 \mathrm{mM}$ Mes (pH 5.5) containing 2 mM EDTA. Four ml of scintillation fluid (Ecolite, Fisher Scientific) were then added with rapid mixing. The resin was allowed to settle and the reaction vials were then counted for radioactivity. Blank control samples contained all reaction components except nitric oxide synthase and were routinely $2 \%-3 \%$ of the added radioactivity. In this assay, unreacted ${ }^{3} \mathrm{H}$-arginine binds to the resin and is completely quenched. Formation of citrulline was calculated from the known specific activity of arginine. Data are presented as the concentration of compound inhibiting iNOS, eNOS or nNOS by $50 \%$.

\section{Acknowledgements}

This work was funded in part by the National Institutes of Health CounterACT Program through the National Institute of Arthritis and Musculoskeletal and Skin Disease (award \# U54AR055073). Its contents are solely the responsibility of the authors and do not necessarily represent the official views of the US federal government.

\section{References}

[1] Laskin, J.D. and Laskin, D.L. (1999) Cellular and Molecular Biology of Nitric Oxide. Marcel Dekker, New York.

[2] Loscalzo, J. (2013) The Identification of Nitric Oxide as Endothelium-Derived Relaxing Factor. Circulation Research, 


\section{3, 100-103. http://dx.doi.org/10.1161/CIRCRESAHA.113.301577}

[3] Rochette, L., Lorin, J., Zeller, M., Guilland, J.-C., Lorgis, L., Cottin, Y. and Vergely, C. (2013) Nitric Oxide Synthase Inhibition and Oxidative Stress in Cardiovascular Diseases: Possible Therapeutic Targets? Pharmacology \& Therapeutics, 140, 239-257. http://dx.doi.org/10.1016/j.pharmthera.2013.07.004

[4] Marletta, M.A. (1993) Nitric Oxide Synthase Structure and Mechanism. Journal of Biological Chemistry, 268, 1223112234.

[5] Kerwin Jr., J.F., Lancaster Jr., J.R. and Feldman, P.L. (1995) Nitric Oxide: A New Paradigm for Second Messengers. Journal of Medicinal Chemistry, 38, 4342-4362. http://dx.doi.org/10.1021/jm00022a001

[6] Stuehr, D.J. and Griffith, O.W. (1992) Mammalian Nitric Oxide Synthases. Advances in Enzymology and Related Areas of Molecular Biology, 65, 287-346.

[7] Förstermann, U., Pollock, J.S., Schmidt, H.H., Heller, M. and Murad, F. (1991) Calmodulin-Dependent EndotheliumDerived Relaxing Factor/Nitric Oxide Synthase Activity Is Present in the Particulate and Cytosolic Fractions of Bovine Aortic Endothelial Cells. Proceedings of the National Academy of Sciences USA, 88, 1788-1792. http://dx.doi.org/10.1073/pnas.88.5.1788

[8] Schmidt, H.H. and Walter, U. (1994) NO at Work. Cell, 78, 919-925. http://dx.doi.org/10.1016/0092-8674(94)90267-4

[9] MacMicking, J., Xie, Q.W. and Nathan, C. (1997) Nitric Oxide and Macrophage Function. Annual Review of Immunology, 15, 323-350. http://dx.doi.org/10.1146/annurev.immunol.15.1.323

[10] Wolff, D.J. and Luneskie, A. (1995) Aminoguanidine Is an Isoform-Selective, Mechanism-Based Inactivator of Nitric Oxide Synthase. Archives of Biochemistry and Biophysics, 316, 290-301. http://dx.doi.org/10.1006/abbi.1995.1040

[11] Malaviya, R., Venosa, A., Hall, L., Gow, A.J., Sinko, P.J., Laskin J.D. and Laskin, D.L. (2012) Attenuation of Acute Nitrogen Mustard-Induced Lung Injury, Inflammation and Fibrogenesis by a Nitric Oxide Synthase Inhibitor. Toxicology and Applied Pharmacology, 265, 279-291. http://dx.doi.org/10.1016/j.taap.2012.08.027

[12] Cinelli, M.A., Li, H., Chreifi, G., Martásek P., Roman, L.J., Poulos, T.L. and Silverman, R.B. (2014) Simplified 2Aminoquinoline-Based Scaffold for Potent and Selective Neuronal Nitric Oxide Synthase Inhibition. Journal of Medicinal Chemistry, 57, 1513-1530. http://dx.doi.org/10.1021/jm401838x

[13] Misra, S., Kuhad, A. and Chopra, K. (2013) Neurobiological Effect of 7-Nitroindazole, a Neuronal Nitric Oxide Synthase Inhibitor, in Experimental Paradigm of Alzheimer's Disease. Indian Journal of Experimental Biology, 51, 10861093.

[14] Silverman, R.B. (2009) Design of Selective Neuronal Nitric Oxide Synthase Inhibitors for the Prevention and Treatment of Neurodegenerative Diseases. Accounts of Chemical Research, 42, 439-451. http://dx.doi.org/10.1021/ar800201v

[15] Marletta, M.A., (1994) Approaches toward Selective Inhibition of Nitric Oxide Synthase. Journal of Medicinal Chemistry, 37, 1899-1907. http://dx.doi.org/10.1021/jm00039a001

[16] Gross, S.S., Stuehr, D.J., Aisaka, K., Jaffe, E.A., Levi, R. and Griffith, O.W. (1990) Macrophage and Endothelial Cell Nitric Oxide Synthesis: Cell-Type Selective Inhibition by $\mathrm{N}^{\mathrm{G}}$-aminoarginine, $\mathrm{N}^{\mathrm{G}}$-nitroarginine and $\mathrm{N}^{\mathrm{G}}$-methylarginine. Biochemical and Biophysical Research Communications, 170, 96-103. http://dx.doi.org/10.1016/0006-291X(90)91245-N

[17] Speltz, L.M., Walworth, B.L. and Pavlista, A.D. (1990) Substituted Nitro and Cyanoguanidines and Their Uses of Increasing Crop Yields. US Patent 4944788.

[18] Arotin, R.L., Walworth, B.L. and Marini, M.E. (1987) US Patent 4639268.

[19] Lutz, A.W. and Rodaway, S.J. (1987) US Patent 4677226.

[20] Casillas, R.P., Kiser, R.C., Truxall, J.A., Singer, A.W., Shumaker, S.M., Niemuth, N.A., Ricketts, K.M., Mitcheltree, L.W., Castrejon, L.R. and Blank, J.A. (2000) Therapeutic Approaches to Dermatotoxicity by Sulfur Mustard I. Modulation of Sulfur Mustard-Induced Cutaneous Injury in the Mouse Ear Vesicant Model. Journal of Applied Toxicology, 20, S145-S151. http://dx.doi.org/10.1002/1099-1263(200012)20:1+<::AID-JAT665>3.0.CO;2-J

[21] Young, S.C., Fabio, K.M., Huang, M.T., Saxena, J., Harman, M.P., Guillon, C.D., Vetrano, A.M., Heck, D.E., Flowers II, R.A., Heindel, N.D. and Laskin, J.D. (2012) Investigation of Anticholinergic and Non-Steroidal Anti-Inflammatory Prodrugs Which Reduce Chemically Induced Skin Inflammation. Journal of Applied Toxicology, 32, 135-141. http://dx.doi.org/10.1002/jat.1645

[22] Jing, Q., Lil, H., Roman, L.J., Martasek, P., Poulos, T.L. and Silverman, R.B. (2014) Accessible Chiral Linker to Enhance Potency and Selectivity of Neuronal Nitric Oxide Synthase Inhibitors. ACS Medicinal Chemistry Letters, 5, 5660. http://dx.doi.org/10.1021/ml400381s

[23] Wolff, D.J. and Datto, G.A. (1992) Identification and Characterization of a Calmodulin-Dependent Nitric Oxide Syn- 
thase from GH3 Pituitary Cells. Biochemical Journal, 285, 201-206.

[24] Wolff, D.J., Mialkowski, K., Richardson, C.F. and Wilson S.R. (2001) C60-Fullerene Monomalonate Adducts Selectively Inactivate Neuronal Nitric Oxide Synthase by Uncoupling the Formation of Reactive Oxygen Intermediates from Nitric Oxide Production. Biochemistry, 40, 37-45. http://dx.doi.org/10.1021/bi0019444 